\title{
Sustained-Exposure Dexamethasone Formulation OTO-104
}

National Cancer Institute

\section{Source}

National Cancer Institute. Sustained-Exposure Dexamethasone Formulation OT 0-104. NCI Thesaurus. Code C132014.

A sustained-release (SR) poloxamer hydrogel formulation containing the glucocorticoid dexamethasone (DXM), with potential otoprotective and anti-apoptotic activities. Prior to the administration of an ototoxic drug and upon intratympanic (IT) injection of the DXM sustained-exposure formulation OT 0-104, DXM is slowly released from the hydrogel over time; DMX then binds to glucocorticoid nuclear receptors, and activates cell survival pathways. This prevents ototoxic drug-induced generation of reactive oxygen species (ROS) by activating anti-oxidant enzymes, blocking the activation of inflammatory cytokines and inducing stress signaling pathways, which ultimately prevents apoptosis of auditory hair cells in the inner ear compartment. This prevents or reduces drug-induced ototoxicity caused by certain therapeutic agents, such as platinum-based anticancer agents. This may reduce or prevent hearing loss, tinnitus, and vertigo. The SR formulation provides increased concentrations of DXM in the inner ear and maintains the elevated levels for a longer time without the need of the multiple IT injections needed when using non-SR DXM formulations. This agent also reduces the symptoms of certain ear-related disorders. 